\title{
TERRENOS CONFIGURADOS ORDENADOS EN LA ALTA MONTAÑA MEDITERRANEA
}

\author{
M. Simón (1), I. Garcia (1), O. Cabezas (1), S. Sánchez (1) \& A. Gómez-Ortiz (2)
}

(1) Departamento de Edafología y Química Agricola. Universidad de Granada.

(2) Departamento de Geografia Fisica y Análisis Geográfico Regional. Universidad de Barcelona.

\begin{abstract}
RESUMEN.- Se estudia el pedón formado bajo un poligono ordenado localizado en la alta montaña mediterránea (Sierra Nevada, España) describiendose y analizándose cuatro perfiles. Los resultados morfológicos y analiticos ponen de relieve que el pedón era muy homogéneo antes de ser deformado por los episodios periglaciares; que la deformación fue desigual sin que supusiera imbriaciones entre horizontes. Estos resultados permiten plantear como hipótesis más probable que la deformación del suelo y la formación de los poligonos de piedras fue resultado de la actuación conjunta de dos tipos de procesos básicos: a) convección térmica del agua; b) empuje ascensional a partir del techo del permafrost.
\end{abstract}

RÉSUMÉ.-On a étudié le "pedon" formé sous un polygone aménagé situé en haute montagne méditerranéenne (Sierra Nevada, Espagne). Pour la caractérisation des sols, on a décrit et analysé quatre profils. Les résultats morphologiques et analytiques mettent en évidence les faits suivants: a) le "pedon" était très homogène avant d'être déformé par des épisodes périglaciaires; b) la déformation a consisté en un élan inégal sur le centre et les bordures du polygone; c) cette déformation n'apas comporté d'imbrications entre les horizons. Ces résultats nous mènent à considérer commel' hypothèse la plus probable l'idée que la déformation du sol et la formation des polygones de pierres ont été causées par l'effet de deux types de processus fondamentaux, agissant ensemble: a) convection thermique de l'eau; b) poussée ascensionnelle à partir de la limite supérieure du permafrost.

ABSTRACT.- The authors study a pedon under a patterned ground, located in a high mediterranean mountain (Sierra Nevada. Spain). Four sedimentological profiles have been described and analyzed. Morphological and analytical results show that the pedon was homogeneous before being modified by several periglacial episodes. It is also possible to observe that the deformation was heterogeneous and it did not imply overlapping between the horizons. These data allow us to conclude that soil deformation and polygonal shaping were the result of both processes: a) thermic convection of water; $b$ ) upward thrust from the permafrost top layer. 
Key words: Patterned ground, Periglaciarisme, Thermic convection, Permafrost, Sierra Nevada.

\section{Introduction}

El término "terreno configurado" fue introducido por Washburn en 1950 para describir "formas más o menos simétricas, tales como círculos, polígonos, redes y estrlas que son características, aunque no necesariamente exclusivas, de mantos sujetos a una intensa acción del hielo". Los polígonos ordenados, forma de terreno configurado analizada en el presente trabajo, fueron definidos por Mattews \& Boyer (1976) como “... terrenos configurados con una morfología predominantemente poligonal y con una apariencia ordenada debida comunmente a bordes de piedras que rodean el material fino". Cuando el material fino del centro del polígono está colonizado por la vegetación, como el descrito en este trabajo, se entiende que se trata de terrenos configurados relictos formados en épocas pasadas más frias.

Si bien existe acuerdo entre la mayoría de los investigadores en cuanto a que la formación de la mayor parte de estos terrenos configurados se asocia a ambientes periglaciares con adecuados ciclos de hielo-deshielo, existen ciertas discrepancias en cuanto a su génesis. Las diferentes hipótesis se pueden agrupar en: a) existencia de grietas, tanto de congelacion como de desecación (Washburn 1979); b) células de convección de Rayleigh (Ray et al., 1983); c) gradiente vertical en la densidad del suelo (Washburn, 1956; Hallet \& Waddington, 1991); d) empuje diferencial por congelación (Washburn, 1956; Van Vliet-Lanoe, 1991) que puede estar favorecido por alguna forma diapírica (Washburn, 1989).

El objetivo del presente trabajo es estudiar los suelos que se forman bajo los terrenos configurados ordenados de la alta montaña mediterránea para, a partir de sus características y propiedades, intentar avanzar en el conocimiento de la génesis de estas peculiares formas periglaciares.

\section{El área de estudio}

El sector donde se desarrollan los terrenos configurados ordenados se localiza en la cabecera del valle de Lanjarón (Sierra Nevada, Granada), a $3100 \mathrm{~m}$ de altitud (Fig. 1). Está enclavado en una antigua superficie de crioplanación, débilmente ondulada ( $2 \%$ de pendiente en dirección SW) y disectada por el desarrollo de los circos glaciares cuaternarios. En él los terrenos configurados ordenados, de tipo flotante, forman polígonos de piedras de diferentes tamaños pero todos ellos alargados en la dirección de máxima inclinación (Gómez Ortiz et al., 1992) (Foto 1). El polígono estudiado tiene un eje transversal medido entre los centros de los bordes de piedras 
TERRENOS CONFIGURADOS ORDENADOS EN LA ALTA MONTAÑA MEDITERRANEA



Figura 1. Esquema del sector occidental de Sierra Nevada y situación geográfica del área de estudio. (En trazo grueso linea de cumbres y en trazo fino red fluvial. La circunferencia delimita la zona de estudio, en la cabecera del valle de Lanjarón) Diagram of the westem sector of Sierra Nevada with the location of the studied area. (Thick line corresponds to mountain ranges and thin line, to fluvial network. The circle delimits the studied area, located in the Lanjaron valley).

de $3.10 \mathrm{~m}$ y un eje longitudinal de $6.40 \mathrm{~m}$ (Fig. 2A). La vegetación del núcleo de estas figuras cerradas está formada por un pastizal con una cobertura del $20 \%$ donde Festuca clementei, Trisetum glaciales y Galium pirenaicum son las especies dominantes (Losa et al., 1976). La precipitación media anual (PMA) es de $1,115 \mathrm{~mm}$ y la temperatura media anual (TMA) de $-1.7^{\circ} \mathrm{C}$, con inviernos muy fríos y relativamente húmedos en los que la temperatura media desciende a $-10.9^{\circ} \mathrm{C}$ y la precipitación representa el $40 \%$ de la PMA, y veranos cálidos y secos en los que la temperatura media asciende a $9^{\circ} \mathrm{C}$ y la precipitación representa únicamenle el $8 \%$ de la PMA. El material original está constituido por micasquistos grafitosos del Manto del Veleta (Díaz de Federico \& Puga, 1976). 




Foto 1. Vista general de un terreno o poligono ordenado General view of an structural polygon.

\section{Material y métodos}

Para abordar este estudio se procedió a abrir una zanja de $5 \mathrm{~m}$ de longitud y $60 \mathrm{~cm}$ de profundidad en dirección tranversal a la máxima pendiente, dejando al descubierto el pedón (Soil Survey Staff, 1975) que se desarrolla bajo los polígonos ordenados (Fig. 2B). A continuación se describieron y muestrearon, para su posterior análisis en el laboratorio, cuatro perfiles de suelos. El perfil 2 (P2) se muestreó en el centro del polígono, los perfiles I y 3 (P1 y $P 3$ ) en los límites internos del borde de piedras y el perfil 4 (P4), en el centro de dicho borde de piedras (Fig. 2B). La descripción y designación de horizontes se hizo de acuerdo con Keys to Soil Taxonomy (Soil Survey Staff, 1990). Todos los datos analíticos están referidos a la tierra fina ( $<2 \mathrm{~mm})$. La textura fue determinada por el método de la pipeta de Robinson. El carbono orgánico se calculó por combustión húmeda con una mezcla $\mathrm{K}_{2} \mathrm{Cr}_{2} \mathrm{O}_{7} / \mathrm{H}_{2} \mathrm{SO}_{4}$ y titulando el dicromato residual con sulfato ferroso (Tyurin, 1951). El pH se, obtuvo potenciométricamente en una suspensión suelo:agua destilada de 1:2.5. La capacidad de cambio catiónico ( $\mathrm{CCC}$ ) se determinó mediante IN $\mathrm{Na}$-acetato a $\mathrm{pH}$ 8.2. Las bases de cambio fueron extraidas con IN NH- 


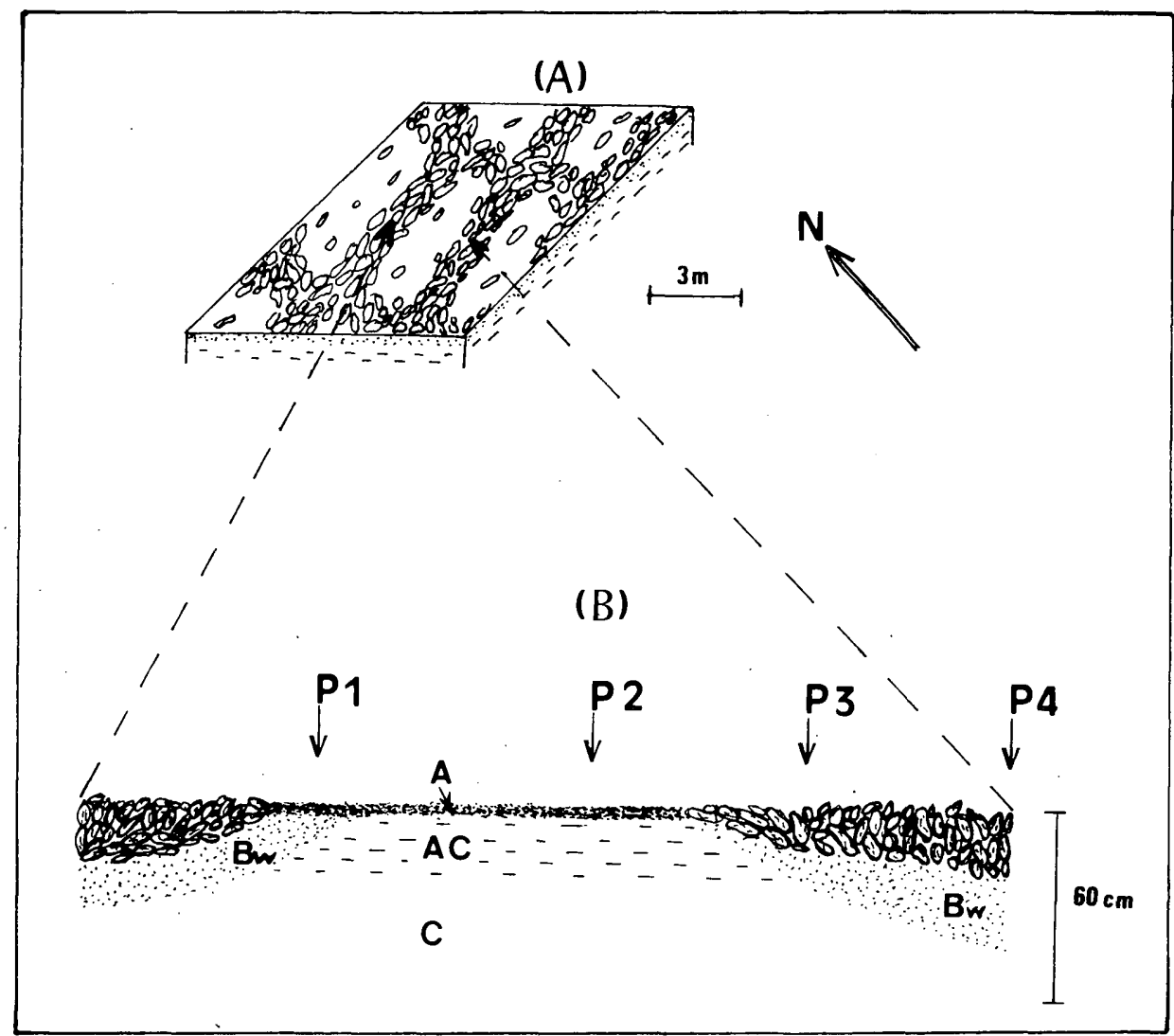

Figura 2. Poligonos ordenados. Aspecto general (A) y detalle del pedón mostrando la disposición de los horizontes y la situación de los cuatro perfiles estudiados (B). Structural polygons. General view (A) and detail of the pedon showing the horizon distribution and the location of the four studied profiles (B).

acetato a pH 7 y determinadas por absorción atómica (Ca y Mg) y fotómetro de llama ( $\mathrm{Na}$ y K). El hierro total (Fet) a través del método de Shapiro \& Brannock (1956). Los óxidos de hierro totales (Fed) fueron extraidos con citrato-ditionito (Holmgren, 1967), el hierro soluble el oxalato (Feo) con oxalato amónico (Schwertmann \& Taylor, 1977) y el hierro soluble en pirofosfato (Fep) con Na-pirofosfato (Bascom, 1968). El hierro de todos los extractos se determinó por absorción atómica. 


\section{Resultados y discusión}

\subsection{Datos de campo de los suelos}

Las características macromorfológicas del pedón ponen de relieve, bajo los bordes de piedras de los poligonos, la presencia de un horizonte Bw con un croma más elevado y una estructura más desarrollada que el horizonte C subyacente (Tabla 1) El límite inferior del horizonte Bw es neto, liso, continuo y regularmente ondulado, con una profundidad máxima en el centro del borde de piedras que disminuye progresivamente hacia el interior del polígono donde llega a desaparecer (Fig.2 B).

Esta morfología evidencia un levantamiento máximo del material del suelo en el centro del polígono y mínimo en los bordes de piedra. Dado que la superficie actual del suelo es completamente plana y que el horizonte BW desaparece del P2, el material del suelo sobreelevado en el centro del polígono debió de sufrir un proceso de erosión o bien un desplazamiento radial hacia los bordes de piedra La presencia en el PI de un horizonte A con características morfológicas muy semejantes a las del mismo horizonte del P2 parece confirmar el desplazamiento radial.

La morfologia del limite inferior del horizonte Bw, continuo y muy bien conservado, sugiere que la deformación del suelo no se llevó a cabo en un estado de elevada plasticidad, que hubiera dado lugar a un límite irregular e incluso interrumpido, sino más bien en condiciones de cierta consistencia o rigidez.

\subsection{Datos analíticos de los suelos}

Los datos analiticos de los horizontes $\mathrm{C}$ de todos los perfiles son muy semejantes entre si (Tabla 2) e indican una gran homogeneidad lateral del material original. En los horizontes suprayacentes los datos analíticos varian en función del desarrollo o no de un horizonte Bw y de la posición que éste ocupe en el pedón.

En todos los horizontes BW se aprecia, en relación a sus respectivos horizontes $\mathrm{C}$, un claro incremento en los valores de arcilla, carbono orgánico, capacidad de cambio, bases de cambio, grado de saturación y pH, que hay que atribuir a la mayor intensidad con que en ellos han actuado los procesos de meteorización y humificación. No obstante, dicho incremento es variable en función del perfil de suelo considerado.

Asi, si se comparan los horizontes BW, el contenido en arcilla y carbono orgánico tiende a disminuir hacia el centro del borde de piedras (P4). Este hecho, unido a que el espesor de dicho borde de piedras es máximo en su centro, se podría interpretar como resultado de un proceso de erosión por las aguas de escorrentía superficial que circularian fundamentalmente por los bordes de piedras de los polígonos y cuya energía sería máxima en el 
TERRENOS CONFIGURADOS ORDENADOS EN LA ALTA MONTAÑA MEDITERRANEA

TABLA 1

Datos de campo de los suelos.

\begin{tabular}{|c|c|c|c|c|c|c|}
\hline Perfil & Horizonte & $\begin{array}{l}\text { Prof. } \\
(\mathrm{cm})\end{array}$ & $\begin{array}{l}\text { Color } \\
\text { seco }\end{array}$ & $\underset{(\%)}{>2 m m}$ & $\begin{array}{l}\text { Estructura } \\
\text { tipo / grado }\end{array}$ & Límite \\
\hline 1 & $\begin{array}{c}A \\
\text { Bw } \\
C\end{array}$ & $\begin{array}{c}0-7 \\
7-31 \\
31-60\end{array}$ & $\begin{array}{c}\text { 10YR 5/3 } \\
7.5 \text { YR } 4 / 4 \\
\text { 10YR } 5 / 2\end{array}$ & $\begin{array}{l}50.5 \\
59.7 \\
47.9\end{array}$ & $\begin{array}{c}\mathrm{f}, \mathrm{m} / \mathrm{d} \\
\mathrm{mf}, \mathrm{bs} / \mathrm{d} \\
/ \mathrm{gs}\end{array}$ & $\begin{array}{l}a, o \\
a, o\end{array}$ \\
\hline 2 & $\begin{array}{c}A \\
A C \\
C\end{array}$ & $\begin{array}{c}0-6 \\
6-20 \\
20-60\end{array}$ & $\begin{array}{l}\text { 10YR } 5 / 3 \\
\text { 10YR } 5 / 2 \\
10 Y R 5 / 2\end{array}$ & $\begin{array}{l}46.6 \\
47.1 \\
48.5\end{array}$ & $\begin{array}{c}f, \mathrm{~m} / \mathrm{d} \\
\mathrm{f}, \mathrm{m} / \mathrm{d}-\mathrm{gs} \\
/ \mathrm{gs}\end{array}$ & $\begin{array}{l}\mathrm{c}, \mathrm{o} \\
\mathrm{g}, \mathrm{o}\end{array}$ \\
\hline 3 & $\begin{array}{l}\text { CP } \\
\text { Bw } \\
\text { C }\end{array}$ & $\begin{array}{c}0-9 \\
9-23 \\
23-60\end{array}$ & $\begin{array}{c}- \\
\text { 7.5YR 4/4 } \\
\text { 10YR 5/2 }\end{array}$ & $\begin{array}{r}100 \\
62.8 \\
48.1\end{array}$ & $\begin{array}{l}\text { mf, bs / d } \\
\text { /gs }\end{array}$ & $\begin{array}{l}a, 0 \\
a, o\end{array}$ \\
\hline 4 & $\begin{array}{l}\mathrm{CP} \\
\mathrm{Bw} \\
\mathrm{C}\end{array}$ & $\begin{array}{c}0-20 \\
20-39 \\
39-60\end{array}$ & $\begin{array}{c}- \\
\text { 7.5YR 4/4 } \\
\text { 10YR 5/2 }\end{array}$ & $\begin{array}{l}100 \\
64.5 \\
48.7\end{array}$ & $\begin{array}{l}\text { mf, bs / d } \\
\text { /gs }\end{array}$ & $\begin{array}{l}a, o \\
a, 0\end{array}$ \\
\hline
\end{tabular}

$\overline{C P}=$ capa de piedras

Estructura: $f=$ fina; $m f=$ muy fina; $m=$ migajosa; $b s=$ sbloques subangulares; $d=$ débil; $g s=$ grano suelto Límite: $\mathrm{a}=$ abrupto; $\mathrm{c}=$ claro; $\mathrm{g}=$ gradual; $\mathrm{o}=$ ondulado

\section{TABla 2}

Datos analiticos de los suelos.

\begin{tabular}{|c|c|c|c|c|c|c|c|c|c|c|c|}
\hline \multirow[b]{3}{*}{ Perfil } & \multirow[b]{3}{*}{ Horizonte } & \multirow{3}{*}{$\begin{array}{l}(\%) \\
\text { O.C. }\end{array}$} & \multirow[b]{3}{*}{$\mathrm{pH}$} & \multicolumn{4}{|c|}{ (cmolc $\mathrm{kg}-1$ ) } & \multirow{3}{*}{$\begin{array}{c}(\%) \\
\text { Satur. } \\
\text { bases }\end{array}$} & \multirow{2}{*}{\multicolumn{3}{|c|}{$<2 \mathrm{~mm} \quad(\%)$}} \\
\hline & & & & \multicolumn{3}{|c|}{ Bases de cambio } & \multirow[b]{2}{*}{$\mathrm{CCC}$} & & & & \\
\hline & & & & $\mathrm{Ca}$ & $\mathrm{Mg}$ & $\mathrm{K}$ & & & Arena & Limo & Arcilla \\
\hline & $\begin{array}{c}A \\
B w \\
C\end{array}$ & $\begin{array}{l}1.48 \\
1.18 \\
0.38\end{array}$ & $\begin{array}{l}5.8 \\
5.6 \\
5.3\end{array}$ & $\begin{array}{l}2.62 \\
2.09 \\
0.35\end{array}$ & $\begin{array}{l}0.45 \\
0.35 \\
0.05\end{array}$ & $\begin{array}{l}0.10 \\
0.07 \\
0.02\end{array}$ & & & $\begin{array}{l}51.9 \\
48.2 \\
52.0\end{array}$ & $\begin{array}{l}36.7 \\
29.9 \\
38.7\end{array}$ & $\begin{array}{r}11.4 \\
21.9 \\
9.3\end{array}$ \\
\hline & $\begin{array}{c}A \\
A C \\
C\end{array}$ & $\begin{array}{l}1.5 \\
0.6 \\
0.4\end{array}$ & $\begin{array}{l}6.0 \\
5.6 \\
5.3\end{array}$ & $\begin{array}{l}3.15 \\
0.94 \\
0.41\end{array}$ & $\begin{array}{l}0.52 \\
0.15 \\
0.08\end{array}$ & $\begin{array}{l}0.02 \\
0.02\end{array}$ & & & $\begin{array}{l}2.0 \\
0.3 \\
9.7\end{array}$ & $\begin{array}{l}36.2 \\
38.5 \\
39.9\end{array}$ & $\begin{array}{l}11.8 \\
11.2 \\
10.4\end{array}$ \\
\hline 3 & $\begin{array}{c}B w \\
C\end{array}$ & $\begin{array}{l}1.10 \\
0.41\end{array}$ & $\begin{array}{l}5.6 \\
5.3\end{array}$ & $\begin{array}{l}1.89 \\
0.38\end{array}$ & $\begin{array}{l}0.29 \\
0.05\end{array}$ & $\begin{array}{l}0.05 \\
0.02\end{array}$ & $\begin{array}{l}8.20 \\
3.37\end{array}$ & $\begin{array}{l}27.2 \\
13.3\end{array}$ & $\begin{array}{l}7.4 \\
2.6\end{array}$ & $\begin{array}{l}24.2 \\
38.2\end{array}$ & $\begin{array}{r}18.4 \\
9.2\end{array}$ \\
\hline & $\begin{array}{c}B w \\
C\end{array}$ & $\begin{array}{l}0.82 \\
0.40\end{array}$ & $\begin{array}{l}5.6 \\
5.3\end{array}$ & $\begin{array}{l}1.56 \\
0.36\end{array}$ & $\begin{array}{l}0.25 \\
0.07\end{array}$ & $\begin{array}{l}0.05 \\
0.02\end{array}$ & $\begin{array}{l}6.91 \\
3.53\end{array}$ & $\begin{array}{l}26.9 \\
12.7\end{array}$ & $\begin{array}{l}60.3 \\
52.1\end{array}$ & $\begin{array}{l}22.3 \\
38.2\end{array}$ & $\begin{array}{r}17.4 \\
9.7\end{array}$ \\
\hline
\end{tabular}

C.O. = carbono orgánico; $\overline{C C C}=$ capacidad de cambio catiónico; Arena $=2-0.2 \mathrm{~mm}$; Limo $=\overline{0.2}-\overline{0.002}$ $\mathrm{mm} ;$ Arcilla $=<0.002 \mathrm{~mm}$ 
centro del borde. En el horizonte Bw delP1 es donde se dan los valores más elevados de cada uno de estos parámetros, lo que unido a que en su superficie parece conservarse parte del material desplazado radialmente desde el centro del polígono (horizonte A), indica que la erosión superficial que sufrió el sector de este perfil fue menor que la que sufrió el sector del perfil P3 y, por supuesto, el del P4.

Semejante morfología y distribución de tamaño de partículas de los distintos horizontes del P2 evidencian que, desde que estos terrenos poligonales dejaron de ser activos hasta la actualidad, la evolución del suelo ha sido muy pequeña. No obstante, la vegetación que lo coloniza y el ciclo biogeoquímico que ésta condiciona, hacen que carbono orgánico, bases de cambio, capacidad de cambio, saturación en bases y $\mathrm{pH}$ aumenten progresivamente hacia la superficie del suelo.

Por otra parte, es importante destacar que los valores de los parámetros anteriores son muy semejantes en los horizontes A de los perfiles PI y P2, lo que reafirmaria el desplazamiento radial de los primeros centímetros del suelo que se sobreelevaron en el centro del polígono.

\subsection{Formas de hierro}

El contenido en hierro total (Fet) es muy parecido en todo los horizontes Cy sistemáticamente tiende a decrecer progresivamente hacia la superficie del suelo (Tabla 3), lo que por un lado confirma la homogeneidad lateral del material original y, por otro, implica una pérdida de hierro (PFet) en los horizontes superficiales con respecto a los subsuperficiales. Según Barahona et al. (1982), esta pérdida de hierro podría ser debida a la disminución del Eh y reducción parcial del hierro durante el deshielo de primavera; no obstante. el valor de PFet en los horizontes Bw se incrementa allf donde la erosión parece ser más intensa (P4), por lo que cabe esperar que parte del hierro se perdiera también por el proceso de erosión de las aguas de escorrentía superficial, bien encerrado en la estructura cristalina de los silicatos o bien en forma de óxidos unidos a la superficie de las arcillas.

En el centro del polígono (P2) las pérdidas de hierro son menores y se producen exclusivamente en el horizonte $A$, pudiéndose atribuir fundamentalmente al deshielo primaveral; por otra parte, son equivalentes a las del horizonte A del P1, lo que vuelve a relacionar ambos horizontes.

Como consecuencia del proceso de meteorización, el total de óxidos de hierro (Fed) se incrementa también en los horizontes Bw en relación a los $\mathrm{C}$ (IFed). Comparando entre sí los horizontes BW, dicho incremento vuelve a ser menor alli donde la erosión superficial parece ser más intensa (P4); por lo que el hierro perdido debería estar fundamentalmente en forma de óxidos.

Si se asume que el material original sobre el que se formó el pedón era vertical y horizontalmente uniforme, tal y como parecen poner de manifiesto las homogeneas caracteristicas morfológicas y analíticas de los distintos 
TERRENOS CONFIGURADOS ORDENADOS EN LA ALTA MONTAÑA MEDITERRANEA

horizontes $\mathrm{C}$, y que el hierro perdido estaba en forma de Fed, se puede estimar la cantidad original de Fed que debieron tener los horizontes BW sumando al valor actual de Fed de cada horizonte Bw el Fet perdido en relación al horizonte $C$ (PFet). Los valores así calculados (Fedc) son muy semejantes en los horizontes Bw de todos los perfiles (Tabla 3) y confirma que el hierro perdido estaba fundamentalmente en forma de Fed.

TABLA 3

Formas de hierro.

\begin{tabular}{|c|c|c|c|c|c|c|c|c|}
\hline Perfil & Horizonte & $\begin{array}{l}\text { Fet } \\
(\%)\end{array}$ & $\begin{array}{l}\text { Fed } \\
(\%)\end{array}$ & $\begin{array}{c}\text { Feo } \\
(\%)\end{array}$ & $\begin{array}{c}\text { Fep } \\
(\%)\end{array}$ & $\begin{array}{c}\text { PFet } \\
(\%)\end{array}$ & $\begin{array}{c}\text { IFed } \\
(\%)\end{array}$ & $\begin{array}{c}\text { Fedo } \\
(\%)\end{array}$ \\
\hline 1 & $\begin{array}{c}A \\
B w \\
C\end{array}$ & $\begin{array}{l}4.40 \\
4.23 \\
4.55\end{array}$ & $\begin{array}{l}1.60 \\
1.80 \\
1.55\end{array}$ & $\begin{array}{l}0.73 \\
0.63 \\
0.39\end{array}$ & $\begin{array}{l}0.23 \\
0.25 \\
0.22\end{array}$ & $\begin{array}{l}0.15 \\
0.32\end{array}$ & 0.25 & $\begin{array}{l}1.75 \\
2.12 \\
1.55\end{array}$ \\
\hline 2 & $\begin{array}{c}A \\
A C \\
C\end{array}$ & $\begin{array}{l}4.41 \\
4.58 \\
4.57\end{array}$ & $\begin{array}{l}1.57 \\
1.55 \\
1.55\end{array}$ & $\begin{array}{l}0.75 \\
0.59 \\
0.40\end{array}$ & $\begin{array}{l}0.22 \\
0.30 \\
0.27\end{array}$ & $\begin{array}{l}0.16 \\
0.00\end{array}$ & 0.00 & $\begin{array}{l}1.73 \\
1.55 \\
1.55\end{array}$ \\
\hline 3 & $\begin{array}{c}\text { Bw } \\
\text { C }\end{array}$ & $\begin{array}{l}4.11 \\
4.56\end{array}$ & $\begin{array}{l}1.72 \\
1.53\end{array}$ & $\begin{array}{l}0.58 \\
0.39\end{array}$ & $\begin{array}{l}0.20 \\
0.22\end{array}$ & 0.45 & 0.19 & $\begin{array}{l}2.17 \\
1.53\end{array}$ \\
\hline 4 & $\begin{array}{c}\text { Bw } \\
\text { C }\end{array}$ & $\begin{array}{l}3.93 \\
4.54\end{array}$ & $\begin{array}{l}1.57 \\
1.50\end{array}$ & $\begin{array}{l}0.42 \\
0.38\end{array}$ & $\begin{array}{l}0.09 \\
0.21\end{array}$ & 0.61 & 0.03 & $\begin{array}{l}2.18 \\
1.50\end{array}$ \\
\hline
\end{tabular}

PFet $=\%$ Fet horizonte C - \% Fet horizontes A, AC o Bw

IFedI $=\%$ Fed horizontes AC o Bw - \% Fed horizonte C

Fedc $=\%$ Fed $+\%$ PFet

Si se comparan las razones Feo:Fed y Fep:Fed en los horizontes Bw y AC (Fig. 3), se aprecia que existe una relación directa entre el valor de las mismas y la intensidad de la erosión superficial, siendo el P4 el que presenta los valores más bajos; por lo que gran parte del Fed perdido estaría en forma de hierro "amorfo" (Feo) y especialmente en su forma ligada a la materia orgánica (Fep). El contenido en carbono orgánico de los diferentes horizontes Bw, tanto menor cuanto mayor es la erosión, apoyaría esta hipótesis. Por otra parte, la erosión provocada por las aguas de escorrentía superficial no parece haber afectado a los horizontes $C$, ya que en ellos los valores de las razones anteriores permanecen prácticamente constantes (Fig.3). 


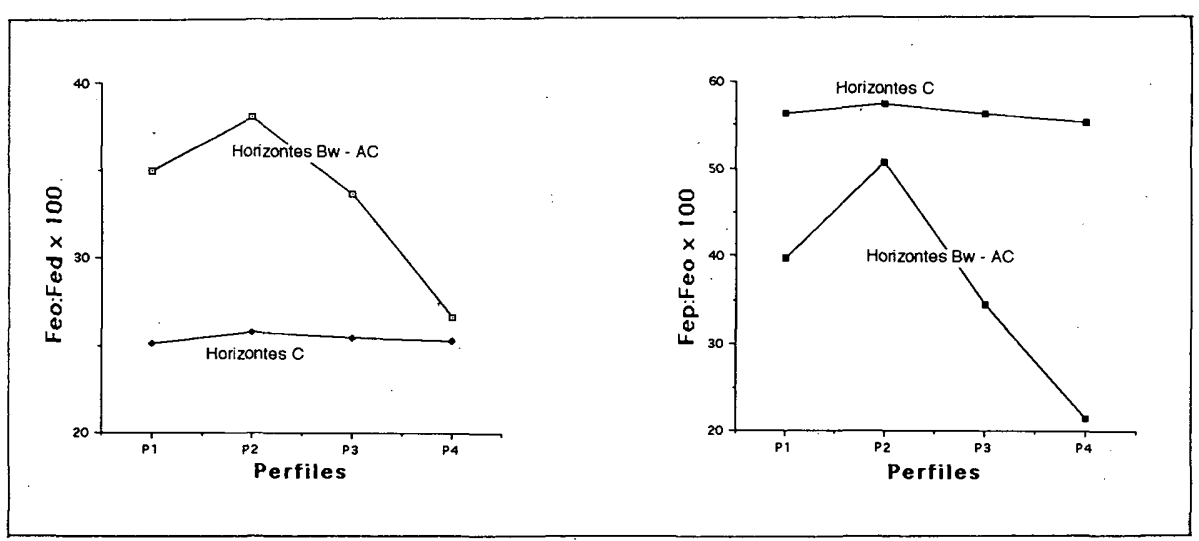

Figura 3. Razones Feo:Fed y Fep:Feo de los horizontes Bw-AC y C. Ratio Feo:Fed and Fep:Feo of the horizons $B W-A C$ and $C$.

\section{Consideraciones generales}

El nivel de evolución equivalente que parecen haber alcanzado los horizontes Bw de los distintos perfiles (valores de Fedc muy semejantes), indica que el pedón, antes de la formación de los polígonos ordenados, era muy homogéneo y debia tener una secuencia de horizontes $A-B w-C$, de características morfológicas y analíticas muy uniformes en todo el sector, con limites planos y relativamente paralelos a la superficie del suelo (Fig. 3A); mientras que los procesos de hielo-deshielo ocurridos en posteriores episodios periglaciares debieron ser los que provocaron su deformación hasta alcanzar la morfología ondulada que actualmente presentan.

Esta morfología evidencia la existencia de un empuje que levantó más, con respecto a la superficie, el suelo situado en el centro del polígono y menos el situado bajo el borde de piedras; hasta el punto de que en el centro del polígono llega a desaparecer el horizonte Bw. Asimismo, dicho empuje deformó el pedón como un todo, conservando perfectamente el límite entre los horizontes; por lo que no parecen haber intervenido, o al menos no lo han hecho de una forma patente, procesos como la convección térmica del suelo o un gradiente vertical de densidad que requieren un estado más o menos fluido del suelo y darlan lugar a mezclas o involuciones que alterarían considerablemente e incluso borrarian la línea base del horizonte Bw. Tampoco parece ser, dada la gruesa textura y la morfologia de la ondulación, con vaguadas más anchas que profundas, que intervengan procesos de agrietamiento del terreno, bien sea por desecación o por congelación. Por tanto, si excluimos estos procesos, el empuje por congelación quedaría 
como el principal responsable de la formación de estos terrenos configurados ordenados.

Con respecto a la distribución de la intensidad del empuje a lo largo del pedón, esta morfología ondulada se podría haber originado a partir de dos tipos de distribución muy diferentes: a) máxima intensidad de empuje en el centro del polígono y mínima en el borde de piedras; y b) intensidad de empuje equivalente a lo largo de todo el pedón pero actuando desde diferentes profundidades, mínima profundidad en el centro del polígono que se iría incrementando progresivamente hasta ser máxima debajo de los bordes de piedras.

La primera hipótesis, máxima intensidad de empuje en el centro del poligono y mínima en el borde de piedras, podría tener su origen en un empuje diferencial por congelación como resultado de la formación de lentes de hielo en el interior del suelo situado en el centro del polígono (horizonte C). La formación de estas lentes de hielo podrían ser debidas a diferencias en la susceptibilidad a la congelación como consecuencia de variaciones espaciales en el contenido en arcilla, limo o materia orgánica (Van Vliet-Lanoe, 1985). No obstante, esta hipótesis tiene dos debilidades fundamentales: la primera es que el horizonte $C$ muestra una gran uniformidad lateral tanto en su textura como en el contenido en materia orgánica, lo que en principio no favoreceria la formación de lentes de hielo; y la segunda, y más importante desde nuestro punto de vista, que el empuje sería máximo en el sector del suelo situado encima de las lentes de hielo y muy bajo o nulo alli donde no existan lentes de hielo, lo que tendería a formar pequeñas fracturas o fallas en el límite entre los horizontes o incluso produciria procesos de crioturbación que borrarian dicho límite.

La segunda hipótesis, intensidad de empuje equivalente a lo largo del pedón pero actuando desde diferentes profundidades, podrla tener su origen en la existencia de un frente de hielo (permafrost) con un límite superior ondulado. Las células de convección de Rayleigh podrian ser la causa que diera lugar a dicha ondulación mediante una desigual transmisión de calor al frente de hielo (Ray et al., 1983). En las zonas donde el agua más caliente y densa penetra en el suelo se produciria una mayor fusión del frente de hielo que daría lugar a la formación de vaguadas, mientras que en las zonas donde asciende el agua más fría y menos densa no existirla fusión o ésta sería mucho menor y se formarían crestas (Fig.4A). A partir de esta morfología ondulada del frente de hielo, la intensidad del empuje por congelacion podría ser muy uniforme en todo el frente y la dirección del empuje perpendicular al mismo (Washburn, 1973); es decir, el empuje sería en ángulo recto a la superficie en el centro de picos y vaguadas e inclinado hacia las vaguadas en los otros sectores (Fig. 4B), lo que implicaría un desplazamiento radial hacia las vaguadas del suelo situado por encima del frente de hielo (Washburn, 1989). De esta forma, el empuje por congelación reproduciria en la superficie del suelo las crestas y las vaguadas de la superficie del frente de hielo, deformando el pedón en su conjunto y 
permitiendo que se conservase perfectamente la línea base del horizonte $\mathrm{Bw}$. En cualquier caso, la convección térmica estaría restringida únicamente al agua (Gleason et al., 1988), ya que si el material del suelo participase en este proceso se produciria una mezcla entre los distintos horizontes y la desaparición de sus límites.

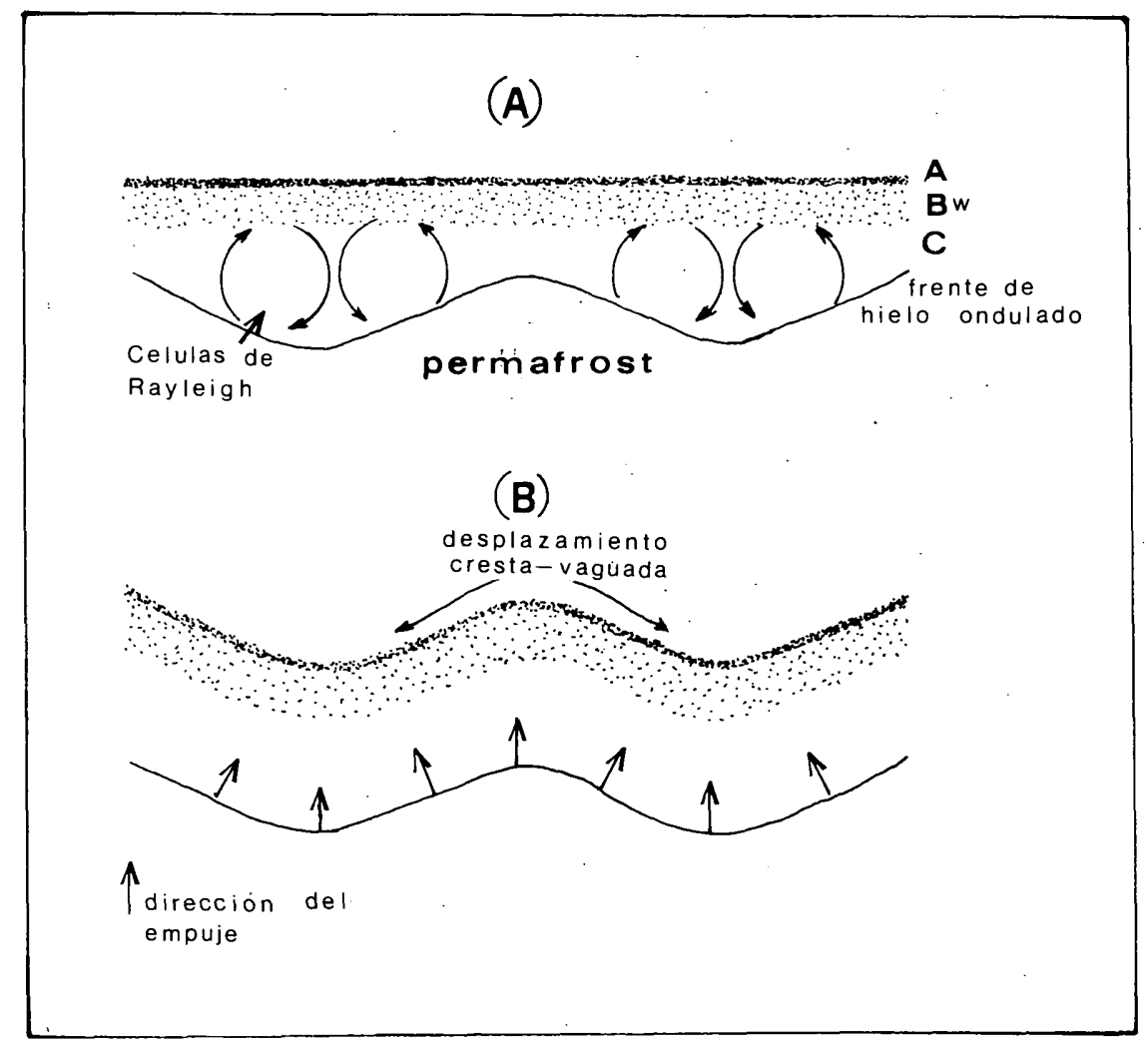

Figura 4. Esquema de las diferentes fases en la hipotética formación de los poligonos ordenados. Diagram showing the several phases of hypothetical development of structural polygons

Esta segunda hipótesis, en la que se combinan las células de convección térmica con el empuje por congelación, se ajusta bien a las características generales, tanto morfológicas como analíticas, del pedón. No obstante, para explicar por completo dichas características es necesario conectarla con otros procesos como: a) inestabilidad gravitacional de los primeros centímetros del suelo de las crestä́s formadas en el centro del polígono y 
desplazamiento radial hacia las vaguadas, que explicaría la desaparición del horizonte BW del P2 y la semejanza existente entre los horizontes A de los perfiles PI y P2; y b) circulación preferencial del agua de escorrentía a traves de las vaguadas, lo que erosionaria completamente el material fino de los primeros centímetros del suelo (borde de piedras) y parcialmente el limo, arcilla, hierro y materia orgánica de los horizontes Bw subyacentes, justificándose así los resultados analíticos de los distintos perfiles del pedón y contribuyendo eficazmente al desarrollo del borde de piedras.

Por otra parte, el borde de piedras y el incremento en el contenido en grava ( $>2 \mathrm{~mm}$ ) de los horizontes Bw en relación a los horizontes $\mathrm{C}$ también podria interpretarse como resultado de que el empuje por congelación, actuando perpendicularmente al frente de hielo, afectase selectivamente a los materiales gruesos en relación a los finos (Corte, 1966) y los desplazase en dirección a las vaguadas. No obstante, dado que los resultados de ambos procesos, erosión por las aguas de escorrentía superficial y empuje selectivo por congelación de los materiales gruesos hacia las vaguadas, serian equivalentes, es muy difícil discernir si los bordes de piedra del polígono son el resultado de un proceso u otro o de la actuación conjunta de los dos, y, en este último caso, cual de ellos actuó con mayor o menor intensidad.

\section{Conclusiones}

La formación de los suelos configurados ordenados en la alta montaña mediterránea parece ser el resultado de la combinación de una serie de procesos y no de un único proceso.

El pedón, originalmente debió de ser morfológica y analíticamente muy homogéneo, con una secuencia de horizontes $A-B W-C$ cuyos límites eran aproximadamente paralelos a la superficie del suelo. Los procesos de hielodeshielo acaecidos en posteriores episodios periglaciares debieron ser los que provocaron su deformación hasta alcanzar la configuración que actualmente presenta.

Esta deformación consistió en una ondulación del terreno en la que las crestas coinciden con el centro de los poligonos y las vaguadas con los bordes de piedras. El empuje que dió lugar a la ondulación afectó al conjunto del pedón como un todo, permitiendo que se conservase perfectamente el límite entre los horizontes.

Estos hechos, unidos al conjunto de las características morfológicas y analíticas de los suelos, nos llevan a considerar, como hipótesis más probable, que su formación pudo ser el resultado de la actuación conjunta de dos procesos fundamentales: convección térmica del agua y empuje por congelación. Otros procesos que se sumarian a los anteriores para configurar los poligonos ordenados, tal y como hoy los observamos, serian un desplazamiento radial del material sobreelevado en el centro del polígono y la erosión del material fino de las vaguadas por las aguas de escorrentía süperficial. 
Agradecimientos. Este trabajo se inscribe en el proyecto "Evolución del paisaje postglaciar de Sierra Nevada. Significación geográfica", de la DGICYT (PS92-0110).

\section{Referenclas}

Barahona, E., Delgado, R. \& Linares, J. (1982): Estudio de las caracteristicas del sistema edáfico de Sierra Nevada mediante análisis factorial. An. Edaf. Agrobiol., 41: 259278.

Bascom, C. L. (1968): Distribution of pyrophosphate extractable iron and organic carbon in soils of various groups. J. Soil Sci., 19: 251-268.

Corte, A. E. (1966): Particle sorting by repeated freezing and thawingl Biuletyn Peryglacjalny, 15: 175-240

Diaz de Federico, A. \& Puga, E: (1976): Estudio geológico del complejo de Sierra Nevada entre los meridianos de Lanjarón y Pitres. Tecniterrae, 9: 1-10.

Gleason, K. J., Krantz, W. B. and Caine, N. (1988): Parametric effects in the filtration free convection model for patterned ground. $V$ Intern. Conf. on Permafrost, Tapir Publishers, 1, 349-354, Trondheim.

Gómez Ortiz, A., Sánchez, S., Simón, M., Salvador, F. \& Esteban, A. (1992): Sintesis de la morfologla glaciar y periglaciar de Sierra Nevada (España). Estudios de Geomorfologia en España, Tomo I, pp. 379-392, Murcia.

Hallet, B. \& Waddington, E.D. (1991): Bouyancy Forces Induced by Freeze-thaw in the Active Layer: Implications for Diapirism and Soil Circulation. In: Dixon, J C. and Abrahams, A. D. (Ed.) Periglacial Geomorphology, John Wiley and Sons Ltd., pp. 251-279, Chichester.

Holmgren, G. (1967): A rapid citrate-dithionite extractable iron procedure. Soil Sci. Am. Proc., 31: 210-211.

Losa, J. M., Molero, J. \& Casares, M. (1986): El paisaje vegetal de Sierra Nevada. La cuenca alta del rio Genil. Serv. Publ. Univ. de Granada, Granada.

Mattews, W. H. \& Boyer, R. E. (1976): Dictionary of geological terms. Anchor Press, New York.

Ray, R. J., Krantz, W. B., Caine, T. N. \& Gunn, R. D. (1983): A model for sorted patternedground regularity. Journal of Glaciology, 102: 317-337

Schwertmann, U. \& Taylor, R. M. (1977): Iron oxides. In: J.B.Dixon and S.B. Webb (Editors), Mineral in Environments. Soil Sci. Soc. Am., pp. 148-180, Madison.

Shapiro, L. \& Brannock, W. (1956): Rapid analysis of silicate rocks. U. S. Geolg Survey Bull., 1036C: 19-56.

Soil Survey Staff (1975): Soil Taxonomy: a basic system of soil classification for making and interpreting soil survey. U. S. D. A. Handbook 436, Washington D.C.

Soil Survey Staff (1990): Keys to Soil Taxonomy. U. S. D. A. SMSS Technical Monograph No.19, Virginia.

Tyurin, I. V. (1951): Analytical procedure for a comparative study of soil humus. Trudy Prochr. Inst. Dokuchaeva, 38: 5-9.

Van Vliet-Lanoe, B. (1985): Frost Effects in soils. In: Boardman, J. (Ed.) Soils and Quaternary Landscape Evolution. Wiley, pp. 115- 156, Chichester.

Van Vliet-Lanoe, B. (1991).: Differential Frost Heave, Load Casting and Convection: Converging Mechanisms; a Discussion of the Origin of Cryoturbations. Permafrost and Periglacial Processes, 2: 123-139.

84 


\section{TERRENOS CONFIGURADOS ORDENADOS EN LA ALTA MONTAÑA MEDITERRANEA}

Washburn, A. L. (1950): Paterned ground. Revue Canadienne de Geographie, 9 (3-4): 5-59.

Washburn, A. L. (1956): Clasification of petterned ground and review of suggested origins. Geol. Soc. Amer. Bull., 67: 823-865.

Washburn, A. L. (1973): Periglacial Processes and Environments. Edward Arnold Publ., 320 pp., London.

Washburn, A. L. (1979): Geocryology: a survey of periglacial processes and environments. Edward Arnold Publ., London.

Washburn, A L. (1989): Near-surface soil displacement in sorted circles, Resolute area Cornwallis Island, Canadian High Arctic. Can J. Earth Sci., 26: 941-955. 